\section{Dental professionals should avoid jargon when speaking to patients}

A dental indemnity body has recommended dental professionals ensure they always use jargon-free, plain English when speaking to patients and colleagues.

In an article ${ }^{1}$ in the Dental Defence Union's (DDU) latest issue of its journal, the union argues that using plain English improves communication with patients but can also help to avoid potential misunderstandings that can lead to complaints or claims.

The Academy of Medical Royal Colleges published guidance ${ }^{2}$ on writing outpatient clinic letters to patients in plain English last year and although it was aimed at medical professionals, the DDU said the advantages applied equally to dentistry.

The Academy guidance explains that it is okay to use some medical jargon but that plain English should be used wherever possible. It recommends explaining acronyms because 'these are often incomprehensible to non-specialists as well as to patients'.

The DDU gives several examples of words commonly used by dental professionals that may not be understood by patients and how they can be explained, including:

- Amalgam - a material commonly used to fill teeth which is silver in colour

- Composite - an alternative filling material which is tooth-coloured

- Restoration - a filling or a crown

- Radiograph - X-ray

- Periodontitis/basic periodontal examination (BPE) - gum disease/a screening test to look for the disease

- Caries - decay in the tooth

- UL5 (or another number) - the notation system used to identify teeth, in this case the fifth tooth back on the upper left of the mouth

- Temporomandibular disorder (TMD) - a condition affecting jaw movement.

Leo Briggs, Deputy Head of DDU, said: 'Jargon, acronyms and technical language are commonly used in dentistry. Because we are using the words day in day out, it can be difficult to distinguish what is and isn't jargon. For example, dental professionals all understand what composite, amalgam and radiographs are, but they are not words widely understood by patients.

'By making the effort to communicate clearly and concisely, dental professionals can give patients a greater sense of involvement in their own care. When you consider that communication issues are also a regular factor in complaints faced by DDU members, using plain English can also minimise the risk of a simple misunderstanding becoming something more serious.

'However, it's not only patients who will benefit from dental professionals adopting a plain English style. Avoiding acronyms and technical language in referral letters and other correspondence with colleagues can also help to avoid misunderstandings and save time in interpretation.'

1. Briggs, L. Plain English for patients. 2018. Available at https://ddujournal.theddu.com/ issue-archive/winter-2018/plain-english-for-patients (accessed January 2019).

2. Academy of Medical Royal Colleges. Please, write to me. Writing outpatient clinic letters to patients. Guidance. 2018. Available at http://www.aomrc.org.uk/reports-guidance/ please-write-to-me-writing-outpatient-clinic-letters-to-patients-guidance/ (accessed January 2019).
BOOK REVIEW

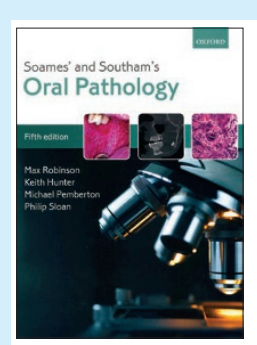

SOAMES' \& SOUTHAM'S ORAL PATHOLOGY, FIFTH EDITION

Max Robinson, Keith Hunter, Michael Pemberton, Philip Sloan

2018; Oxford University Press; price $£ 46.99$ pp. 256 ISBN: 9780199697786

New to print, this fifth edition of Soames' and Southam's classic text on oral pathology has been completely revised by experts in the field of oral pathology and oral medicine. More than ten years has passed since the last update and so the content has been modernised to address advancements in diagnostic techniques and evidence-based management guidelines for today's clinical practice. The textbook is available in paperback and e-book format, and is designed to meet the educational needs of both the modern dental professional in training, and existing clinicians, who may require a reference manual in surgery.

As with previous editions, the opening chapter deals with key principles of clinical examination and assessment, the formation of a differential diagnosis and selection of further investigations such as laboratory testing. Subsequent chapters guide the reader through diseases of the oral mucosa, including oral cancer and other potentially malignant disorders; ideal for current practitioners wanting to refresh their knowledge of this core continuing professional development topic. The remaining chapters are arranged in a logical manner, with diseases of the salivary glands; diseases of teeth and supporting structures; jaw cysts and odontogenic tumours and bone disorders.

This edition also features two all new chapters, with 'skin diseases affecting the oro-facial region' describing important lesions that clinicians should recognise on the face and lips. The other new chapter covers the differential diagnosis of neck lumps, describing pathology which is frequently encountered in the dental surgery. The final chapter discusses oral manifestations of systemic disease, and may be of particular interest to undergraduates sitting the human diseases elements of their course.

Overall, this new edition places more emphasis on commonly encountered oral diseases. Detailed descriptions of pathology which is now less prevalent have been removed and new information on diseases more likely to be encountered today has been added. Content of chapters where topics are better dealt with in other texts; such as dental caries, periodontal diseases, temporomandibular joint disorders, and oral diseases in children has similarly been reduced. As in previous editions, handy key points boxes and summary tables are utilised throughout to emphasise important areas, and these provide an excellent aid to learning and revision.

The result is a relatively concise text, at almost half the size of similar publications in the field, which retains its status as a comprehensive guide to oral pathology. It will undoubtedly help form a sound and reliable knowledge base for dental undergraduate study, while remaining an easily accessible read for clinicians wanting to refresh their knowledge of key aspects of clinical oral medicine.

Richard Leck 